\title{
LIMIT SETS OF AUTOMORPHISM GROUPS OF A TREE
}

\author{
SU-SHING CHEN
}

\begin{abstract}
Let $X$ be an infinite and locally finite tree and $G$ be an arbitrary automorphism group of $X$. The limit set $L(G)$ of $G$ in the boundary $B$ of $X$ is studied.
\end{abstract}

1. Introduction. A tree is a nonempty connected graph $X$ without circuit. One can associate a tree to $S L_{2}$ over the field $Q_{p}$ as a special case of the Bruhat-Tits building which is the $p$-adic analogue of homogeneous symmetric spaces of real semisimple Lie groups [1], [2], [7], [8]. Other applications are the graph $\Gamma(G, P)$ of a group $G$ and a subset $P$ of $G$ and algebraic geometry [7]. Automorphism groups and harmonic analysis on a tree have been investigated by Tits [8] and Cartier [1], [2] respectively.

In the theory of hyperbolic plane and fuchsian groups one has the definition of the limit set $L(G)$ of a subgroup $G \subseteq S L_{2}(R)$. This concept will be defined for an arbitray automorphism group $G$ on an infinite and locally finite tree $X$. We shall obtain some general facts about $L(G)$ such as the cardinality of $L(G)$ and the relative size of $L(G)$ in the boundary $B$ of $X$. In [5] and [6], Kulkarni has considered this question for a general class of topological spaces. He has also investigated the connection with the theory of ends of Freudenthal and Hopf. Our main result is a theorem similar to that of Ahlfors for Kleinian groups [3].

2. Tree. Let $X$ be an infinite and locally finite tree with vertex set $S$ and edge set $A$. Let $s$ and $t$ be vertices and be connected. Then the edge $\{s, t\}$ is in $A$. (For simplicity we do not emphasize orientation here.) $\mathrm{A}$ path in $X$ is a sequence $C=\left[s_{0}, s_{1}, \ldots, s_{n}\right]$ of vertices such that $s_{i-1}$ is connected to $s_{i}$ for $1<i<n$. The length of $C$ is $n$. If all the vertices in a path $C_{0}$ are distinct, then this unique path $C_{0}$ is called a geodesic. The length of the geodesic joining vertices $s$ to $t$ is called the distance $d(s, t)$ between them. Let $s$ be a vertex. The infinite path originated from $s$ is an infinite sequence $C=\left[s_{0}, s_{1}, \ldots, s_{n} \ldots\right]$ of vertices such that $s_{0}=s$ and $s_{n}$ and $s_{n+1}$ are connected for each $n>0$. $C$ is called an infinite geodesic if $s_{i} \neq s_{j}$ for $i \neq j$. The relation that two infinite geodesics $C$ and $C^{\prime}$ have infinitely many common vertices is an equivalence relation. Each equivalence class is called an end. The set $B$ of all ends is the boundary to $X$. We can join each $s$ in $S$ and each $b$ in $B$ by an infinite geodesic. Moreover, each pair $b \neq b^{\prime}$ in $B$ can be joined by an infinite geodesic. With the topology described in Theorem 1.1 of [2], the space

Received by the editors October 31, 1979 and, in revised form, February 19, 1981. 1980 Mathematics Subject Classification. Primary 05C05, 05C25; Secondary 22E40. 
$\hat{S}=S \cup B$ is a compact and totally disconnected space such that $S$, with the discrete topology, is an open dense subspace of $\hat{S}$. Let $s$ and $s^{\prime}$ be two vertices. If the vertex $t$ moves along the infinite geodesic representing the end $b$, then $d(s, t)-d\left(s^{\prime}, t\right)$ is a finite constant $\delta_{b}\left(s, s^{\prime}\right)$. A horocycle $H$ through $s$ is the set of vertices $s^{\prime}$ such that $\delta_{b}\left(s, s^{\prime}\right)=0$. There is a partition of $S$ into a disjoint union of horocycles $\left\{H_{n}\right\}_{n=0}^{\infty}$.

Let $g$ be an automorphism of $X$. Tits [8] has proved that one of the following holds:

(1) there exists $s$ in $S$ such that $g(s)=s$,

(2) there exist $s$ and $s^{\prime}$ in $S$ such that $\left\{s, s^{\prime}\right\}$ is an edge and $g(s)=s^{\prime}$ and $g\left(s^{\prime}\right)=s$,

(3) there exist $\left\{s_{n}\right\}_{n=-\infty}^{\infty}$ such that $\left\{s_{n} s_{n+1}\right\}$ is an edge and $g\left(s_{n}\right)=s_{n+k}$ for any $n$ and some $k>0$.

Furthermore, he has proved that if $G$ is an automorphism group of $X$ and $G$ has no element of type (3), then $G$ leaves a vertex $s$, an edge $\left\{s, s^{\prime}\right\}$ or an end $b$ (together with all horocycles associated to $b$ ) invariant. It is obvious that automorphisms of $X$ are isometries with respect to the distance $d\left(s, s^{\prime}\right)$ defined above. Each automorphism $g$ of $X$ can be extended to act on the boundary $B$. The action is a homeomorphism.

3. Limit set. With the topology on $\hat{S}=S \cup B$, we define the limit set $L(G)$ of an automorphism group $G$ to be the set of accumulation points in $B$ of an orbit $G s$ of $s$. That is $L(G)=\overline{G s} \cap B$. There is the question as to whether $L(G)$ is independent of the choice of the vertex $s$. Let $s$ and $s^{\prime}$ be two vertices with finite distance $d\left(s, s^{\prime}\right)=d$. We have two orbits $G s$ and $G s^{\prime}$. Each $b$ in $L(G)$ is the limit $\lim g_{n} s$ for some sequence $g_{n}$ in $G$. Let $b^{\prime}$ be the $\operatorname{limit} \lim g_{n} s^{\prime}$. Since $d\left(s, s^{\prime}\right)=d\left(g_{n} s, g_{n} s^{\prime}\right)=d$ and $d\left(b, b^{\prime}\right)=\infty$, we have $b=b^{\prime}$. Consequently, $L(G)$ is independent of the choice of $s$.

Proposition 1. (i) $L(G)$ is a closed invariant subset of $B$ under $G$.

(ii) Let $A$ be any closed invariant subset of $B$ under $G$ and let $A$ contain more than one point. Then $A \supset L(G)$ and $L(G)$ is minimal under the action of $G$ on $B$.

(iii) The set of fixed points of elements of type (3) (if they exist) is dense in $L(G)$.

Proof. It is clear that $L(G)$ is closed. The invariance of $L(G)$ is seen by $b=\lim g g_{n}(s)=g \lim g_{n}(s)=g(b)$. To prove (ii), let $b$ and $b^{\prime}$ be in $A$. Let $b^{\prime \prime}$ be in $L(G)$ such that $\lim g_{n} s=b^{\prime \prime}$. The two infinite points $b$ and $b^{\prime}$ can be joined by an infinite geodesic. We choose $s$ to be on that geodesic. Then either $g_{n} b$ or $g_{n} b^{\prime}$ must converge to $b^{\prime \prime}$. Thus $A \supset L(G)$. (iii) follows from (ii) directly. In fact, each element of type (3) has two fixed points in $B$. The closure of fixed points of elements of type (3) in $G$ can be taken as $A$.

Proposition 2. The cardinality of $L(G)$ of an automorphism group $G$ is $0,1,2$ or $\infty$.

PRoof. Either $G$ contains no elements of type (3) or it does contain them. In the first case, $G$ has a fixed point $s$ or an invariant edge $\left\{s, s^{\prime}\right\}$ or a fixed end $b$. If $G$ 
has a fixed point $s$ or an invariant edge $\left\{s, s^{\prime}\right\}$, then $L(G)$ is empty. If $G$ has a fixed end $b$, then $G$ leaves horocycles associated to $b$ invariant.

The orbit $G s$ will converge to $b$, if we take $s$ to be a vertex on a horocycle associated to $b$. The limit set $L(G)$ consists of one point. In the second case, $L(G)$ contains at least two points, because two ends $b$ and $b^{\prime}$ of the infinite geodesic translated by an element $g$ of type (3) will be limit points. If $L(G)$ has more than two points, then $L(G)$ has infinitely many points. In fact, if $L(G)$ has finitely many points, then the $n$th power $g^{n}$ of any element $g$ of $G$ will leave every point in $L(G)$ fixed when $n$ is sufficiently large. Then $g^{n}$ has at least two fixed ends and must translate the infinite geodesic joining them. Thus $g$ is of type (3) and $L(G)$ can have only two points.

REMARK. We may obtain a classification (see Corollary 3.5 of [8] also) of automorphism groups on an infinite and locally finite tree $X$. There are the following possibilities:

(i) $G$ has a common fixed point $s$ in $S$ or has a common invariant edge $\{s, t\}$ in A. $L(G)$ is empty.

(ii) $G$ has a common fixed end $b$ in $B$ and $L(G)$ consists of one point.

(iii) $G$ has two common fixed ends $b_{1}$ and $b_{2}$ in $B$ and $L(G)$ consists of two points.

(iv) $G$ has an invariant proper subtree $X^{\prime}$ (not a vertex, an edge or an infinite geodesic) of $X$ and $L(G)$ is the boundary $B^{\prime}$ of $X^{\prime}$.

(v) $G$ acts minimally on $B$ and $L(G)=B$.

From the above, we also obtain a classification of quotient graphs $G \backslash X$ of $X$ if $G$ acts freely on $X$. There are the following possibilities:

(i) $G \backslash X$ is parabolic if $L(G)$ has one point and $G \backslash X$ is a union of graphs $G \backslash H_{n}$, which are the quotient graphs of horocycles $H_{n}$ by $G$.

(ii) $G \backslash X$ is axial if $L(G)$ consists of two points. There is an infinite geodesic $C$ which is translated by $G$ and $G \backslash C$ is the unique circuit such that the injection $G \backslash C \rightarrow G \backslash X$ is a homotopy equivalence [7].

(iii) $G \backslash X$ is fuchsian if $L(G)$ consists of infinitely many points. This is the most interesting case and offers a great variety of quotient graphs.

4. Boundary measure. We use $\$ \S 2.5,3.3$ and 3.4 of [2] to investigate the boundary measure of $L(G)$ of an automorphism group $G$. We refer to [1], [2] for details. Let $X$ be an infinite and locally finite tree. For each finite path $a$, we assign a positive number $p(a)$ such that $p\left(a a^{\prime}\right)=p(a) p\left(a^{\prime}\right)$ if the composite path $a a^{\prime}$ is defined, and for $s, s^{\prime} \in S, \sum p\left(s s^{\prime}\right)=1$ for all $s^{\prime}$ connected to $s$. If $h$ is a function on $S$, we define the function $P h$ on $S$ by $P h(s)=\sum p\left(s s^{\prime}\right) h\left(s^{\prime}\right)$ over the set of all vertices $s^{\prime}$ connected to $s$. Let $\Delta h=P h-h$ be the Laplace operator and let $K(s, b)$ be the Martin kernel of $X$. There exists a positive measure $\mu$ of mass 1 on $B$ such that

$$
\int_{B} K(s, b) \mu(d b)=1
$$

for each $s$ in $S$. This measure is called the Poisson measure. Consider a random walk $\left\{X_{0}, X_{1}, \ldots, X_{n}, \ldots\right\}$ on $S$ in the following manner. If $X_{0}=0$ is the original point and $X_{n}=s$, then the permissible values of $X_{n+1}$ are vertices $s^{\prime}$ connected to $s$ 
with probability $p\left(s, s^{\prime}\right)$ from $s$ to $s^{\prime}$. By the Borel-Cantelli lemma, $X_{n}$ converges to a point $X_{\infty}$ in $B$ with probability 1, with respect to the probability law given by the Poisson measure $\mu$ on $B$. If $h$ is a bounded harmonic function, the Martingale theorem shows that the sequence of random variables $\left\{h\left(X_{n}\right)\right\}$ converges to a random variable $Y$ with probability 1 . There exists a bounded Borel function $\phi$ on $B$ such that $Y=\phi\left(X_{\infty}\right)$ and one has the Poisson integral formula

$$
h(s)=\int_{B} K(s, b) \phi(b) \mu(d b), \quad \text { any } s \in S .
$$

The radial convergence theorem of Fatou holds in the following sense: $\phi(b)=$ $\lim h\left(s_{n}\right)$, almost everywhere in $B$ with respect to $\mu$, on an infinite geodesic $\left[s_{0}, s_{1}, \ldots, s_{n}, \ldots\right]$ joining the original point 0 to $b$.

A limit point $b$ in $L(G)$ is said to be an approximation limit point if for any sequence $\left\{s_{n}\right\}$ in a geodesic approaching to $b$, there exists a sequence $\left\{g_{n}\right\}$ in $G$ such that $g_{n}\left(s_{n}\right)$ lies in a finite set (compact set). This notion is originally due to Hedlund ([3], [6] for further references). Because $g_{n}\left(s_{n}\right)$ lies in a finite set, without loss of generality we may assume that they are all equal. Thus, we can find a sequence $\left\{s_{k}\right\}$ converging to $b$ and $\left\{g_{k}\right\} \subseteq G$ such that $\left\{g_{k}\left(s_{k}\right)\right\}$ is a point in $S$. It is obvious that fixed points of elements of type (3) are approximation limit points. Also the set $A(G)$ of approximation limit points of $G$ is $G$-invariant.

TheOREM. Let $X$ be an infinite and locally finite tree and $G$ be an automorphism group of $X$. Then the Poisson measure $\mu(A(G))$ of the set $A(G)$ of approximation limit points of $G$ is either 0 or 1 . If $L(G)$ contains almost all approximation limit points, then the Poisson measure $\mu(L(G))$ is either 0 or 1 .

Proof. The characteristic function $\phi$ on $B$ of $A(G)$ is $G$-invariant and takes values 0 and 1 . Let $h$ be the harmonic function

$$
h(s)=\int_{B} K(s, b) \phi(b) \mu(d b) .
$$

Then $h$ is also $G$-invariant. If the measure $\mu(A(G))$ is positive, then there is a point $b$ in $A(G)$ such that Fatou's convergence theorem can be applied. There exists an infinite geodesic $C$ approaching $b$ such that the radial limit of $h(s)$ along $C$ approaching $b$ must exist and is equal to 1 . Note that $0<h(s)<1$ for all $s$ in $S$. Since each $b$ in $A(G)$ is an approximation limit point, there is a sequence $\left\{s_{n}\right\}$ on $C$ converging to $b$ and there is a sequence $\left\{g_{n}\right\}$ in $G$ such that $g_{n}\left(s_{n}\right)$ is a point $s_{0}$ in $S$. Then the $G$-invariance and continuity implies that $h\left(s_{0}\right)=1$. But 1 is the upper bound for $h(s)$. Hence $h(s)$ attains its maximum at an interior point $s_{0}$ in $S$. $h(s)$ has to be the constant 1 and

$$
\begin{aligned}
\mu(A(G)) & =\int_{A(G)} K(s, b) \mu(d b) \\
& =\int_{B} K(s, b) \phi(b) \mu(d b) \quad(\phi \text { is the characteristic function) } \\
& =\int_{B} K(s, b) \mu(d b) \quad\left(\text { since } \phi(b)=\lim _{s \rightarrow b} h(s)=1\right)=1
\end{aligned}
$$


If $L(G)$ contains almost all approximation limit points (that is, except a set of measure zero), then we have $\mu(L(G))$ is 0 to 1 by the above result of $A(G)$.

\section{REFERENCES}

1. P. Cartier, Géométrie et analyse sur les arbres, Séminaire Bourbaki, 1971/72, Lecture Notes in Math., vol. 317, Springer-Verlag, Berlin and New York, 1973, pp. 123-140.

2. __ Fonctions harmoniques sur un arbre, Symposia Math. 9 (1972), 203-270.

3. S. Chen, A theorem of Ahlfors for hyperbolic spaces, Trans. Amer. Math. Soc. 242 (1978), 401-406.

4. P. Eberlein and B. O'Neill, Visibility manifolds, Pacific J. Math. 46 (1973), 45-109.

5. R. Kulkarni, Groups with domains of discontinuity, Math. Ann. 237 (1978), 253-272.

6. S_ Some topological aspects of Kleinian groups, Amer. J. Math. 100 (1978), 897-911.

7. J. Serre, Arbres, amalgames, $S L_{2}$, Soc. Math. France Astérique, No. 46, 1977.

8. J. Tits, Sur le groupe des automorphismes d'un arbre, Essays on Topology and Related Topics, Mémoires dédiés á G. de Rham, Springer-Verlag, Berlin and New York, 1970, pp. 188-211.

Department of Mathematics, University of Florida, Gannesville, Florma 32611 